\title{
Percepção materna sobre qualidade de vida de crianças portadoras de cárie na primeira infância: um estudo piloto
}

Maternal perception of the quality of life of children with early childhood caries: a pilot study

Percepción materna de la calidad de vida de los niños con caries de la primera infancia: un estudio piloto

\author{
Vlamir Oliveira da SILVA ${ }^{1}$ \\ Marcelle DANELON ${ }^{2}$ \\ Marília Andrade Figueiredo de OLIVEIRA ${ }^{3}$ \\ Nathalia Cristina TAVELLA E SILVA ${ }^{4}$ \\ Eliana Mendes de Souza Teixeira ROQUE ${ }^{5}$
}

${ }^{I}$ PhD, Departamento de Odontologia, Universidade de Ribeirão Preto, UNAERP, 14096-900 Ribeirão Preto - SP, Brasil

${ }^{2}$ PhD, Departamento de Odontologia, Universidade de Ribeirão Preto, UNAERP, 14096-900 Ribeirão Preto-SP, Brasil,

Departamento de Odontologia Preventiva e Restauradora, Faculdade de Odontologia, UNESP Univ. Estadual Paulista, 16015-050 Araçatuba - SP, Brasil

${ }^{3}$ Graduanda, Departamento de Odontologia, Universidade de Ribeirão Preto, UNAERP, 14096-900 Ribeirão Preto - SP, Brasil

${ }^{4} M$ s, Departamento de Odontologia, Universidade de Ribeirão Preto, UNAERP, 14096-900 Ribeirão Preto-SP, Brasil

${ }^{5}$ PhD, Departamento de Odontologia, Universidade de Ribeirão Preto, UNAERP, 14096-900 Ribeirão Preto - SP, Brasil

\section{Resumo}

Objetivo: O objetivo deste estudo foi compreender a percepção materna sobre a qualidade de vida de crianças, portadoras de cárie na primeira infância (CPI). Material e Método: O objeto de estudo foram seis mães de crianças em tratamento na Clínica de Odontologia da Universidade de Ribeirão Preto. Realizou-se entrevista semi-estruturada gravada. Os dados foram analisados segundo a técnica de Análise de Conteúdo proposta por Bardin e utilizando-se os conceitos teóricos oriundos de Bronfembrenner para avaliar aspectos da CPI que interferem no pleno desenvolvimento da pessoa desde a infância. Depreendeu-se dos dados, 03 categorias empíricas: 1 - "o dente natural é tudo"; $2^{\mathrm{a}}$ - " Procura o dentista quando tem dor" e $3^{a}$ - "A mãe tem responsabilidade no aparecimento da cárie porque não limpa a boca da criança". Resultados: Os resultados demonstraram que a influência dos aspectos sócio-econômicos e educacionais das mães tornam as crianças mais vulneráveis à CPI. As mães compreendem a importância dos dentes na saúde bucal, porém, não atribuem a presença da CPI a predispor as crianças ao desenvolvimento de problemas sistêmicos e afetar o desenvolvimento biopsicossocial. As mães apontaram falhas no atendimento das crianças e de acesso ao serviço público, assim como, o despreparo dos profissionais do serviço público e privado. Conclusão: Conclui-se que que existe necessidade de ampliação de medidas socioeducativas tanto para mães quanto crianças, assim como estratégias que visem formar e ampliar o conhecimento coletivo em saúde bucal e melhoria da Política Nacional da Saúde Bucal.

Descritores: Comportamento Materno; Qualidade de Vida, Cárie Dentária; Saúde da Criança.

\section{Abstract}

Objective: The objective of this study was to understand the maternal perception of the quality of life of children with early childhood caries (IPC). Material and Method: The object of the study was six mothers of children under treatment at the Dental Clinic of the University of Ribeirão Preto. A semi-structured recorded interview was carried out. The data were analyzed according to the Content Analysis technique proposed by Bardin and using the theoretical concepts from Bronfembrenner to evaluate aspects of CPI that interfere in the full development of the person since childhood. The data revealed 03 empirical categories: 1st - "the natural tooth is everything"; 2nd - "Look for the dentist when he has pain" and 3rd - "The mother has responsibility in the appearance of caries because she does not clean the child's mouth". Results: The results showed that the influence of the socio-economic and educational aspects of mothers make children more vulnerable to CPI. Mothers understand the importance of teeth in oral health, but do not attribute the presence of CPI to predispose children to the development of systemic problems and affect biopsychosocial development. Mothers pointed out failures in child care and access to public service, as well as the unpreparedness of public and private service professionals. Conclusion: It is concluded that there is a need to expand social and educational measures for both mothers and children, as well as strategies that aim to form and expand collective knowledge in oral health and improve the National Oral Health Policy.

Descriptors: Maternal Perception; Quality of Life; Dental Caries; Child Health.

\section{Resumen}

Objetivo: El objetivo de este estudio fue entender la percepción materna de la calidad de vida de los niños con caries de la primera infancia (IPC). Material y método: El objeto del estudio fueron seis madres de niños en tratamiento en la Clínica Dental de la Universidad de Ribeirão Preto. Se realizó una entrevista grabada semiestructurada. Los datos fueron analizados de acuerdo con la técnica de Análisis de Contenido propuesta por Bardin y utilizando los conceptos teóricos de Bronfembrenner para evaluar los aspectos de la IPC que interfieren en el desarrollo completo de la persona desde la infancia. Los datos revelaron 03 categorías empíricas: $1^{\text {a }}$ - "el diente natural lo es todo"; $2^{\text {a }}$ "Busque al dentista cuando tenga dolor" y $3^{\mathrm{a}}$ - "La madre tiene la responsabilidad en la aparición de la caries porque no limpia la boca del niño". Resultados: Los resultados mostraron que la influencia de los aspectos socioeconómicos y educativos de las madres hacen que los niños sean más vulnerables al IPC. Las madres entienden la importancia de los dientes en la salud bucal, pero no atribuyen la presencia de IPC a la predisposición de los niños al desarrollo de problemas sistémicos y afectan al desarrollo biopsicosocial. Las madres señalaron las fallas en el cuidado de los niños y en el acceso al servicio público, así como la falta de preparación de los profesionales de los servicios públicos y privados. Conclusión: Se concluye que es necesario ampliar las medidas sociales y educativas tanto para las madres como para los niños, así como las estrategias destinadas a formar y ampliar el conocimiento colectivo en materia de salud bucal y mejorar la Política Nacional de Salud Bucal.

Descriptores: Percepción Materna; Calidad de Vida; Caries Dental; Salud del Niño.

\section{INTRODUÇÃO}

\begin{tabular}{lrr}
\hline A cárie dentária continua & sendo uma das \\
grandes & preocupações da & Odontologia
\end{tabular}
contemporânea, tendo permanência elevada na faixa etária pediátrica ${ }^{1}$. De acordo com a American Academy of Pediatric Dentistry (AAPD), em um amplo estudo de revisão literária, a cárie na primeira infância é "a presença de uma ou mais superfícies deterioradas (não cavitadas ou cavitadas), ausentes (devido à cárie) ou preenchidas em qualquer dente primário de uma criança entre o nascimento e os 71 meses"

De acordo com os dados da Pesquisa Nacional de Saúde Bucal brasileiro, em 2010, uma criança brasileira possui, em média, o índice de 2,43 dentes com experiência de cárie. Em 2016, segundo o Levantamento Epidemiológico Nacional Brasileiro 
em saúde bucal observou prevalência de $26,85 \%$ de experiência de cárie em crianças entre 18 e 36 meses com aumento desta prevalência com o avanço da idade $^{3-5}$.

Em um amplo estudo de revisão literária sobre os fatores causais da Cárie na Primeira Infância, verificou-se que a etiologia da cárie está fortemente associada à higiene bucal deficiente, hábitos alimentares inadequados e transmissibilidade dos microrganismos cariogênicos. Ademais, outros fatores foram relacionados, como condições socioeconômicas, culturais, psicológicas e comportamentais. A abordagem precoce da saúde bucal na criança é de suma importância, visto o impacto que promove no decorrer da vida. Logo, a prevenção deve ser priorizada mais cedo possível, através de abordagem multiprofissional, repercutindo na saúde bucal das crianças e de todo núcleo familiar ${ }^{6}$.

Com isso, nos despertamos a entender como as mães de crianças portadoras de cárie na primeira infância interpretam esta situação de seu filho, se ela tem percepção do quanto à cárie pode afetar o desenvolvimento biopsicossocial e a qualidade de vida de seu filho.

\section{MATERIAL E MÉTODO}

Para a compreensão do objeto de estudo, foi optado a análise qualitativa, considerada como aquela desenvolvida no estudo da história, das relações, das crenças, das representações e das opiniões resultantes das interpretações que os indivíduos realizam a respeito do modo como vivem, sentem, pensam, além de mostrar processos sociais ainda pouco conhecidos relativos a grupos particulares, dando a oportunidade de construir novas abordagens e estabelecer novos conceitos e categorias ${ }^{7}$.

A amostra foi constituída de 6 mães de crianças portadoras de cárie precoce da infância em atendimento na Clínica de Odontopediatria da Universidade de Ribeirão Preto (UNAERP). Este número de mães se justifica, pois, as últimas entrevistas apresentaram prévias ao estudo piloto, apresentaram saturação dos dados.

A coleta de dados foi realizada através de entrevistas semiestruturadas que foram gravadas, transcritas e analisadas. As mães dos participantes do trabalho foram previamente selecionadas após a avaliação das crianças pela anamnese e exame clínico que indicaram serem portadoras de cárie precoce da infância. Após assinarem o Termo de Consentimento Livre e Esclarecido, as mães forneceram sua identificação e dados socioeconômicos com vistas a contemplar seu perfil familiar. As mesmas foram identificadas pela sílaba $\mathrm{M}$ seguida de números ordinais em ordem crescente a fim de manter o anonimato.
As questões norteadoras foram: (1) "Qual é seu entendimento sobre a função dos dentes numa pessoa? E quanto ao seu filho, como você percebe a saúde dos dentes dele? Fale-me um pouco sobre isso?"; (2) "Você entende que os dentes estragados do seu filho podem acarretar problemas atuais e futuros para ele? Se positivo, quais seriam estes problemas e de que maneira poderiam atrapalhar a vida dele como um todo? Se negativo, por que não?"; (3) "Na sua percepção por qual motivo os dentes e a boca do seu filho necessitaram a vinda ao dentista, tem alguma explicação? Você se sente responsável pela atual condição bucal de seu filho? Comente a sua resposta positiva ou negativa.".

Para análise dos dados, utilizamos a Análise de Conteúdo, que segundo $\operatorname{Bardin}^{8}$ abrange as iniciativas de explicitação, sistematização e expressão do conteúdo de mensagens, com a finalidade de se efetuarem deduções lógicas e justificadas a respeito da origem dessas mensagens (quem as emitiu, em que contexto e/ou quais efeitos se pretende causar por meio delas).

Para responder aos nossos objetivos e contextualmente avaliar os aspectos da cárie na primeira infância que interferem no pleno desenvolvimento da pessoa desde a infância, utilizamos os conceitos oriundos de Alves ${ }^{9}$. Para esta autora, pessoa e contexto não podem ser separados, pois ambos são detentores de variáveis, com diferentes e múltiplos níveis. É o chamado modelo bioecológico, que tende a reforçar a ênfase nas características biopsicológicas da pessoa em desenvolvimento ${ }^{10}$. No modelo bioecológico, são reapresentados quatro aspectos multidirecionais interrelacionados: "pessoa, processo, contexto e tempo".

O projeto deste estudo foi aprovado pelo Comitê de Ética e Pesquisa da Universidade de Ribeirão Preto (CAAE: 48542015.0.00005498).

\section{RESULTADOS}

\section{- Caracterização dos participantes}

O perfil socioeconômico e demográfico das mães de crianças que apresentam cárie na primeira infância está ilustrado nas Tabelas 1 e 2 , e permite constatar que essas mães pertencem a um nível socioeconômico das camadas populares e a atividade econômica exercida por elas correspondem a atividades técnicas e manuais. Todas as mães apresentam no máximo, o ensino médio completo.

- Compreensão da percepção materna sobre qualidade de vida de seus filhos portadores de cárie na primeira infância

$\mathrm{Na}$ análise das entrevistas depreendemos 3 categorias empíricas apresentadas a seguir:

\section{"O dente natural é tudo"}

As mães entrevistadas ao referenciarem que "o dente natural é tudo" acenam para a importância de totalidade que dão à questão dentária, denotando 
que embora valorem desta forma, não atribuem o mesmo sentido para os aspectos gerais de saúde, que se apresenta nas falas dissociadas de tal entendimento, como pode ser percebido no discurso de M1, com 23 anos de idade, ensino fundamental completo e vida laborativa como auxiliar de limpeza.

MI - O dente natural é tudo, serve melhor que a dentadura, a dentadura pra mastigar, tem que tirar, o dente normal não. É tudo numa pessoa o dente, .... Quando ela vai comer dependendo do tipo de comida ela reclama que sente dor.

Outras mães, como M2, de apenas 19 anos e com menor renda entre as entrevistadas, e também M5, mãe mais adulta com 32 anos de idade e renda familiar melhor que M2, confirmam as falas de M1:

M2: "Pra saúde, eu acho que a gente também né, não sei muito ..., porque ela não deixava escovar, não deixava fazer nada, agora depois que veio pra cá melhorou, ela com, ela não comia carne, ela come bastante coisa, escovar ela tá deixando, e melhorou bastante, ela não reclama mais de dor, tá bem melhor."

M5: "Tudo. Pra mastigar, ingerir os alimentos, né. Os dentes têm que estar bons e fortes. Os dentes dele começou a atrapalhar a alimentação dele, quando ele tava com as cárie, porque doía muito."

$\mathrm{Na}$ fala destas mães, há demonstração do entendimento da importância dos dentes. Acham fundamental a presença dos dentes $\mathrm{e}$ fazem reiterações sobre sua utilidade na alimentação e, no caso da cárie e da dor, dificultarem a vida da criança. Porém, não percebem a influência dos dentes na vida psicológica, social e na saúde sistêmica da criança.

Já M4, destaca em sua fala a relevância dos dentes na alimentação e no sorriso de seu filho.

M4- Eu acho que serve assim, para tudo né. Pra ter um sorriso melhor, pra comer e.....muda o rosto da gente, né, que o sorriso fica melhor com os dentes na boca.

Tal observação também foi compartilhada por M3, mãe de 33 anos e com maior renda.

M3 - Para mastigar bem os alimentos, ter um sorriso bonito .... Mas as cáries dela vêm muito rápido. Enfim, você faz o tratamento e daqui a pouco está com cárie de novo.

$\mathrm{O}$ fator estético impulsionou as mães a procurarem o dentista, porém, sem considerar os fatores psicológicos e físicos influenciados pela presença da cárie e falta de dentes, como a dificuldade na alimentação que leva a desnutrição tornando-a mais susceptível a outras doenças e, além disso, a aparência comprometida provocar baixo rendimento escolar (vergonha, bulling) e diminuição da autoestima. Outra observação importante está na frase de M3 "a cárie vem muito rápido" que não desconfia que a cárie se desenvolve dessa forma, devido à má higiene da criança.

Tais aspectos estão bem retratados nas falas das mães quando questionadas sobre problemas futuros provocada pela cárie precoce da infância, como pontuado pelas mães M2, M4 e M5.

M2: Não, assim não, estou sabendo agora que vim pra cá, que to se informando sobre os dentes, porque antes eu não sabia.

M4: Sim, eu já ouvi falar que traz problema pro coração, porque eu já assisti uma palestra. Tem outra doença lá também, mas foi o do coração que eu guardei mais.

M5: Sim, mas não sei.

Consideramos que apesar de entenderem que a saúde bucal é tudo, as mães se preocupam com problemas provocados pela presença da cárie de mamadeira, voltados para mastigação ou estética, mas desconhecem efeitos no desenvolvimento biopsicossocial de seus filhos.

Tabela 1. Perfil socioeconômico e demográfico das mães das crianças portadoras de cárie na primeira infância

\begin{tabular}{c|c|c|c|c|c}
\hline Mãe & $\begin{array}{c}\text { Idade } \\
\text { da mãe }\end{array}$ & Escolaridade & Profissão & Estado civil & Cor \\
\hline M1 & 23 & Fundamental & $\begin{array}{c}\text { Auxiliar de } \\
\text { limpeza }\end{array}$ & União estável & Parda \\
\hline M2 & 19 & Fundamental & Manicure & Casada & Branca \\
\hline M3 & 33 & Ensino Médio & $\begin{array}{c}\text { Auxiliar de } \\
\text { enfermagem }\end{array}$ & Casada & Parda \\
\hline M4 & 32 & $\begin{array}{c}\text { Fundamental. } \\
\text { Incompleto }\end{array}$ & Faxineira & Solteira & Parda \\
\hline M5 & 32 & Ensino Médio & Artesã & Casada & Branca \\
\hline M6 & 26 & Fundamental & Do lar & Casada & Parda \\
\hline
\end{tabular}

Tabela 2. Perfil socioeconômico e demográfico das famílias das crianças portadoras de cárie na primeira infância

\begin{tabular}{c|c|c|c}
\hline $\begin{array}{c}\text { Renda familiar } \\
(\mathbf{R} \$)\end{array}$ & $\begin{array}{c}\text { Responsável } \\
\text { sustento da casa }\end{array}$ & $\begin{array}{c}\text { Número de } \\
\text { filhos }\end{array}$ & $\begin{array}{c}\text { Ordem de } \\
\text { nascimento }\end{array}$ \\
\hline 2.300 & Casal & 01 & $1^{\mathrm{o}}$ \\
\hline 800 & Casal & 01 & $1^{\mathrm{o}}$ \\
\hline 5.200 & Casal & 02 & $2^{\mathrm{o}}$ \\
\hline 880,00 & A própria & 04 & $4^{\mathrm{o}}$ \\
\hline 2.500 & Marido & 03 & $3^{\mathrm{o}}$ \\
\hline 2.500 & Marido & 02 & $2^{\mathrm{a}}$ \\
& & &
\end{tabular}

\section{"Procura o dentista quando tem dor"}

As mães revelaram haver necessidade da dor para procurar por atendimento. Existe desconhecimento sobre o desenvolvimento da doença cárie ou dos procedimentos preventivos. $\mathrm{O}$ que se revela na fala das mães M3 E M5:

M3: Dores, não tá podendo comer direito, beber água, líquidos gelados ou até mesmo quente, sorriso, se ela for querer sorrir ela não vai, vai ter vergonha de estar sorrindo. Pra mim é isso.

M5- Dor né, procurei mais porque tava sentindo muita dor, ele chorava muito de dor, dai a gente procurou, porque já estava atrapalhando ele em tudo. Beber leite, comer, brincar, .... daí veio o desespero mesmo e fui procurar ajuda.

A falta de acesso ao serviço público ou desconhecimento dos dentistas em relação à cárie na primeira infância, também foi relatado, e até mesmo, falhas nas orientações preventivas e do atendimento odontológico, transparecendo haver falta de vontade do profissional em resolver o problema agravando a situação das crianças. 
Estas observações são constatadas nas falas de M1, M4 e M6:

M1: "É a cárie que começou né... os dentes dela tava no começo, nascendo, já com problema, eu levei no dentista, só que a dentista não insistiu em fazer um tratamento forçado com ela, ai quando foi encaminhar praqui já era um pouco tarde, já tava mais avançado."

M4: "Então ele ficou daquele jeito ali... daí o dente já tava quebrado, dai ele quebrou e ficou daquele jeito ali. Ai fui pra dentista de Santo Antonio e mandou pra cá. E não tinha mais jeito assim lá."

M6: "Se tivesse pegado uns dentistas bom acho que não teria arrancado os dentes dela. Quando começou a dar um pouquinho de cárie, eu levei, e eles falou: “ mãe é dente de leite e logo vai cair, então não tem problema". Ai foi indo, começou a ficar podre, eu paguei particular, e foi a mesma coisa: "ai mãe é dente de leite e logo vai cair"... ai até que eu consegui a vaga aqui. Só que daí eu consegui depois que caíram os 4 dentes. Já tava feio. Levei desde pequenininha".

"A mãe tem responsabilidade no aparecimento da cárie porque não limpa a boca da criança".

Mães entrevistadas demonstraram culpa pelo aparecimento da cárie de mamadeira, como observado na fala de M3 e M4.

M3: "Pra mim, todo dentista fala que é a mamadeira, porque a gente escova o dente dela direitinho, tá fazendo tudo sempre certinho e sempre sai a cárie. Então, pra gente é a mamadeira noturna, porque ela já dorme com a mamadeira na boca .... Com certeza, a culpa é minha né. Eu tinha que tentar fazer com que ela não desse essa mamada ou se mamasse, ela escovasse o dente dela, só que eu fico com dó de acordar, e eu me sinto muito mal, porque ela reclama de dor."

M4: Eu acho que é assim, mamadeira, antibiótico né. Que causou.

Responsabilidade: A gente né, porque, quando sempre é de menor a gente é responsável. Né. Quando ele tiver de maior, daí ele responde pra cima.

Ao conhecer o fator etiológico causador da cárie dentária, as mães se sentem culpadas, tanto pelo desconhecimento como pelo sentimento de dó em obrigar o filho a realizar ações de prevenção como a simples escovação dos dentes, como reforça a fala das mães M2 e M5.

M2: "Ah, ela comia bastante doce e não deixava escovar, ...não gostava de escovar e eu deixava, acabava deixando... E agora eu faço, nem se ela chorar eu faço ela escovar. No começo eu sentia (responsável), porque ela não escovava, agora melhorou, mas antes eu achava que era."

M5: Responsabilidade: "Sim, eu acho que a gente mãe é muito cômodo, igual ele, mamava no peito, eu não limpava a boca dele direto, sabe, aí quando queria, deixava, é mais fácil pra gente ir levando. Eu só caí em si depois que eu vi a cárie na boca dele. Hoje se ele estiver dormindo eu acordo ele pra limpar os dentinho dele. Quer dizer, a gente tem que ter conhecimento pra passar pra ele, porque ele depende de mim, né. Então o erro é meu, eu acho que o erro é meu."

No relato dessas mães, nota-se que, à medida que adquiriram o conhecimento, houve uma mudança da postura e hábitos, e o sentimento da responsabilidade supera o de condoimento.

DISCUSSÃO

O termo "cárie na primeira infância" corresponde ao acometimento pelas lesões em crianças de até 6 anos de vida, originada devido a hábitos alimentares inadequados, higiene oral insatisfatória, dente outros fatores considerados como secundários ${ }^{2,11}$. Consiste em um quadro grave, agudo e doloroso que acomete crianças em idade pré-escolar e induz os responsáveis a procurarem um profissional da saúde, principalmente, devido à sensação dolorosa consequente quando não tratada 12 . O acometimento dessas lesões varia de país, como demostrado por Lai et al. ${ }^{13}$ sendo $27,9 \%$ na Inglaterra, $40 \%$ nos Estados Unidos, $50 \%$ no Japão, 48,7 na Austrália e 46\% no Brasi $^{14,15}$, relatando também o comprometimento da estética, fonação, mastigação e deglutição, causando dificuldade na alimentação e perda de peso, prejudicando o crescimento, promovendo a redução do desempenho escolar e afetando o desenvolvimento da criança, isso é, influenciando na qualidade de vida da criança ${ }^{11,13,16-18}$

Na família, ganha destaque a figura da mãe, por ser o membro com voz decisória nas questões de saúde e doença, sendo seu papel essencial para construção de uma saúde bucal satisfatória da criança e, por consequência da família, sendo responsável pelo equilíbrio no binômio saúde-doença ${ }^{19}$. A amostra utilizada neste estudo foi composta por 6 mães entre 19 e 33 anos, cujos filhos estavam em atendimento na Clínica de Odontopediatria da Universidade de Ribeirão Preto (UNAERP), previamente selecionadas após avaliação das crianças pela anamnese e exame clínico que indicaram serem portadoras de cárie precoce da infância. Os resultados encontrados neste estudo permitem constatar que as mães entrevistadas tem perfil sócio econômico e nível de escolaridade diferentes, demostrando não haver relação com o surgimento da cárie precoce na infância, concordando com o estudo de Vollú et al. ${ }^{12}$ e Zaror et al. ${ }^{20}$ que demonstraram que a cárie na primeira infância é consequência do consumo de açúcar e má higienização da cavidade oral e não diretamente dos determinantes sociais de saúde, que são a renda, escolaridade, ocupação e etnia, como são 
influenciadas as enfermidades transmissíveis e a desnutrição ${ }^{15}$.

Fejerskov e Kidd $^{21}$ relatam que o desenvolvimento da cárie é resultado de um processo dinâmico, ou seja, de uma dissolução química provocada pela atividade metabólica em um depósito microbiano que cobre a superfície do dente resultando em um desequilíbrio mineral e subsequente formação de lesão de cárie. As mães entrevistadas relatam entenderem que a saúde bucal de seus filhos seja importante e que pode ocasionar problemas futuros, porém desconhecem sobre $\mathrm{o}$ desenvolvimento e prevenção da doença cárie e a importância da higiene oral de seus filhos assim como observado por Ferreira et al. ${ }^{22}$, que os pais apresentam conhecimento limitado sobre a saúde bucal de seus filhos, e destacaram a necessidade de abordar pontos referentes à etiologia e transmissão da cárie dentária, uso de dentifrícios fluoretados e higiene bucal concluindo ser imprescindível que pais e cuidadores sejam orientados para 0 desenvolvimento de correta higienização prevenção.

São considerados também como fatores secundários de risco a cárie dentária, a quantidade de membros da família, seus conhecimentos relacionados à saúde bucal e sua renda ${ }^{23}$. As mães entrevistadas relataram possuir entre 01 a 04 filhos, sendo seus filhos mais novos acometidos à cárie, além de possuir um companheiro que é responsável parte ou integralmente pelo sustento da família, com exceção da M4, que seu estado civil é solteiro. Todas relataram que consideram os dentes como de grande importância para a criança, porém, não associaram a influência da saúde bucal com a saúde sistêmica corroborando com Mansoori et al. ${ }^{24}$ que destacaram que, muitas pessoas consideram as doenças bucais sem importância por não acarretarem risco de morte. No entanto, as alterações na cavidade bucal podem gerar um alto impacto na qualidade de vida e Batliner et al. ${ }^{25}$ observaram que a saúde para as mães reveste de um significado amplo, não restrito ao biológico. A saúde bucal encontra-se dissociada da saúde geral e as práticas de manutenção da saúde bucal são voltadas para a prevenção de uma única doença: a cárie, relacionando sua ocorrência na dependência dos cuidados prestados por elas. As mães representam a ideia de que a saúde bucal tem seu espaço de menor valor frente a outros mais importantes e determinantes no seu modo de vida ${ }^{24}$.

Diante dos relatos das mães entrevistadas, observou-se que a estética foi importante para impulsionar as mães a levarem seus filhos ao dentista e não a dificuldade em alimentar-se, o baixo rendimento escolar e a influencia na qualidade de vida, como também observado por BaniHani et al. ${ }^{16} \mathrm{e}$ Jiang et al. ${ }^{11}$ que as mães não apresentam percepção sobre a influência da condição dental de seus filhos nas suas condições biopsicológicas, a principal importância dada aos dentes se relaciona à estética.

Além da estética, foi relatado que as mães procuraram o atendimento odontológico quando seus filhos apresentaram sensação dolorosa nos dentes, sem citar a busca por prevenção, podendo isto ser resultado de falta de informação desconhecimento da etiologia, desenvolvimento e prevenção da doença cárie como Phantumvanit et al. ${ }^{19}$ ressaltaram, que as mães desconhecem a época de levar seus filhos à primeira consulta, ignorando sobre o aparecimento de cáries na dentição decídua, só buscando tratamento de emergência devido à dor e problemas estéticos.

As mães também relataram dificuldades no acesso ao atendimento odontológico, tanto público como particular, e também desconhecimento do dentista sobre o atendimento de seus filhos, contribuindo para o agravamento dos casos. As causas e consequências da cárie na primeira infância são bastante conhecidas pelos profissionais, demonstrando que são capacitados a orientar os cuidadores, porém muitos desses não são capacitados para realizar procedimentos ocasionando iatrogenias e consequente agravamento do caso ${ }^{19}$.

Durante a entrevista, as mães demonstraram se sentirem culpadas pela condição bucal das crianças, seja por desconhecimento ou pelo sentimento de pena em obrigar seus filhos a escovarem seus dentes. As mães têm uma cobrança significante em relação a essa obrigação social, sendo apontadas como responsáveis pelas condições de saúde bucal dos seus filhos ${ }^{19}$. Com isso, torna-se válido investir em atenção e orientação em saúde bucal aos responsáveis pelas crianças que ao ter acesso a informações são motivadas a adoção de hábitos saudáveis e consequente redução da cárie dental como demostrado no estudo de Vollú et al. ${ }^{12}$ que destacaram que as mães assumem plenamente, a tarefa de responsáveis pela saúde bucal de seus filhos. Os relatos de mães de famílias sobre saúde e os meios de verificar e garantir a saúde da sua família refletem, sobretudo, sua vivência do papel de zelar por aqueles que estão sob sua responsabilidade e, que a mãe atua como agente multiplicador de informações, sendo crucial para a criação e manutenção do elo família-profissional.

Quando coexistem envolvimentos dos fatores sociais e biológicos existe a importância em informar para responsabilizar, dessa forma as cobranças e aconselhamentos sobre saúde bucal pelos profissionais da saúde com empenho e sem hostilidade, rompendo barreiras impostas e proporcionando a população a conscientização para a superação dos limites e a busca pela qualidade de vida $^{11,13,16-18}$.

\section{CONCLUSÃO}

Com base na metodologia empregada e nos 
resultados obtidos concluiu-se que existe necessidade de ampliação de medidas socioeducativas tanto para mães quanto crianças, assim como estratégias que visem formar e ampliar o conhecimento coletivo em saúde bucal e melhoria da Política Nacional da Saúde Bucal.

\section{REFERÊNCIAS}

1. Calcagnile F, Pietrunti D, Pranno N, Di Giorgio G, Ottolenghi L, Vozza I. Oral health knowledge in pre-school children: A survey among parents in central Italy. J Clin Exp Dent. 2019:11:e327-33.

2. Chase I, Berkowitz RJ, Proskin HM, Weinstein P BR. Clinical outcomes for Early Childhood Caries (ECC): the influence of health locus of control. Eur J Paediatr Dent. 2004;2:76-80.

3. Brasil Ministério da Saúde. Projeto SB Brasil 2003 Condições de saúde bucal da população brasileira2002-2003.http://cfo.org.br/wpcontent/uploads /2009/10/04_0347_M.pdf. Published 2004. Accessed October 4, 2009.

4. Brasil Ministério da Saúde. SB Brasil 2010: Pesquisa Nacional de Saúde Bucal. http://bvsms.saude.gov.br/bvs/publicacoes/pesquis a_nacional_saude_bucal.pdf. Published 2012. Accessed October 4, 2009.

5. Guedes-Pinto AC. Odontopediatria. 9th ed. (Santos, ed.); 2016.

6. Macedo LZ, Ammari MM. Cárie da primeira infância: conhecer para prevenir. Rev Rede Cuid em Saúde.2014;8:1-14.

7. Minayo MC de S. O Desafio Do Conhecimento: Pesquisa Qualitativa Em Saúde. 1st ed. (Hucitec, ed.); 2012.

8. Bardin L. Análise de Conteúdo. 70th ed. (France PU de, ed.). Lisboa; 1977.

9. Alves PB. A ecologia do desenvolvimento humano: experimentos naturais e planejados. Psicol Reflexão e Crítica. 1997;10:369-73.

10. Bronfenbrenner, U., \& Morris PA. The ecology of developmental processes. In: Inc. JW\& S, ed. Handbook of Child Psychology: Theoretical Models of Human Development. v. 1. New York; 1998:993-1028.

11. Jiang M, Wong MCM, Chu CH, Dai L, Lo ECM. Effects of restoring SDF-treated and untreated dentine caries lesions on parental satisfaction and oral health related quality of life of preschool children. J Dent. 2019;88:103171.

12. Vollú AL, da Costa M da EPR, Maia LC, Fonseca-Gonçalves A. Evaluation of Oral HealthRelated Quality of Life to Assess Dental Treatment in Preschool Children with Early Childhood Caries: A Preliminary Study. J Clin Pediatr Dent.2018;42:37-44.

13. Lai SHF, Wong MLW, Wong HM, McGrath CPJ, Yiu CKY. Factors influencing the oral healthrelated quality of life among children with severe early childhood caries in Hong Kong.Int J Dent Hyg.2019;17:350-58.

14. Abanto J, Carvalho TS, Mendes FM, Wanderley MT, Bönecker M, Raggio DP. Impact of oral diseases and disorders on oral health-related quality of life of preschool children. Community Dent Oral Epidemiol. 2011;39:105-14.

15. American Academy of Pediatric Dentistry. Guidelines for Policy on Social Determinants of Children's Oral Health and Health Disparities. Am Acad Pediatr Dent. 40:23-26.

16. BaniHani A, Deery C, Toumba J, Munyombwe T, Duggal M. The impact of dental caries and its treatment by conventional or biological approaches on the oral health-related quality of life of children and carers. Int J Paediatr Dent. 2018;28:266-76.

17. Knorst JK, Menegazzo GR, Emmanuelli B, Mendes FM, Ardenghi TM. Effect of neighborhood and individual social capital in early childhood on oral health-related quality of life: a 7-year cohort study. Qual Life Res.2019;28:1773-82.

18. Marshman Z, Knapp R. Child oral health-related quality of life following treatment under dental general anaesthetic (DGA). Evid Based Dent. 2019;20:46-7.

19. Phantumvanit P, Makino Y, Ogawa $\mathrm{H}$, et al. WHO Global Consultation on Public Health Intervention against Early Childhood Caries. Community Dent Oral Epidemiol.2018;46:280-87.

20. Zaror C, Atala-Acevedo C, Espinoza-Espinoza G, et al. Cross-cultural adaptation and psychometric evaluation of the early childhood oral health impact scale (ECOHIS) in chilean population. Health Qual Life Outcomes. 2018;16:232.

21. Kidd E, Fejerskov O. Changing concepts in cariology: forty years on. Dent Update. 2013;40:277-86.

22. Ferreira JMS, Silva FS, Aragão AKR, Duarte RC, Menezes VA. Conhecimento de pais sobre saúde bucal na primeira infância. Pediatr Mod.2010;46:224-30.

23. Novaes TF, Pontes LRA, Freitas JG, et al. Responsiveness of the Early Childhood Oral Health Impact Scale (ECOHIS) is related to dental treatment complexity. Health Qual Life Outcomes.2017;15:182.

24. Mansoori S, Mehta A, Ansari MI. Factors associated with Oral Health Related Quality of Life of children with severe -Early Childhood Caries. J Oral Biol Craniofacial Res.2019;9:222225.

25. Batliner $\mathrm{T}$, Fehringer KA, Tiwari $\mathrm{T}$, et al. Motivational interviewing with American Indian mothers to prevent early childhood caries: study design and methodology of a randomized control trial. Trials. 2014;15:125. 


\section{CONFLITO DE INTERESSES}

Os autores declaram não haver conflitos de interesse.

\section{AUTOR PARA CORRESPONDÊNCIA}

\section{Marcelle Danelon}

Universidade de Ribeirão Preto (UNAERP)

Departamento de Odontologia

Av. Costábile Romano 2201

14096-900 Ribeirão Preto - SP, Brasil

Tel. +55 16 3603-7000

Universidade Estadual Paulista (UNESP)

Faculdade de Odontologia de Araçatuba

Departamento de Odontologia Preventiva e Restauradora

Rua José Bonifácio 1193

16015-050 Araçatuba - SP, Brasil

Tel. +551836363235

Email: marcelledanelon@ @otmail.com

Submetido em 31/03/2020

Aceito em 04/06/2020 